\title{
Base Metal Complexes as Homogeneous Catalysts and Enzyme Mimics
}

\author{
Xile $\mathrm{Hu}^{*}$
}

\begin{abstract}
This article is a short overview of some recent research activity in the Laboratory of Inorganic Synthesis and Catalysis (LSCI) at EPFL-ISIC. It summarizes the work on Ni-catalyzed cross-coupling reactions of non-activated alkyl halides. It then describes and discusses the work on the bio-mimetic chemistry of [Fe]-hydrogenase.
\end{abstract}

Keywords: Bio-inorganic chemistry · [Fe]-hydrogenase · Homogeneous catalysis · Organometallic chemistry · Nickel

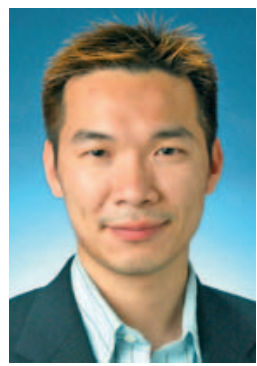

\section{Introduction}

I joined the Institute of Chemical Sciences and Engineering (ISIC) at EPFL as a tenure-track assistant professor in summer 2007. The mission of my research group is to develop catalysts made of earth-abundant elements for synthesis, energy, and sustainability. Upon my arrival in Lausanne, we started two new projects. The first one is on Ni-catalyzed cross-coupling reactions and the second one is on the model chemistry of [Fe]-hydrogenase. And just recently, we embarked on a project to develop bio-inspired catalysts for hydrogen evolution.

\section{Ni-catalysis}

Our work on $\mathrm{Ni}$ catalysis is part of the program to develop well-defined coordination complexes of late first-row transition metal ions as catalysts for fine chemical synthesis. Our starting point is the pincer-type bis(amino)amide ligand ${ }^{\mathrm{Me}} \mathrm{N}_{2} \mathrm{~N}$ (Scheme 1). Pincer ligands are

${ }^{\star}$ Correspondence: Prof. Dr. X. L. Hu

Ecole Polytechnique Fédérale de Lausanne Institute of Chemical Sciences and Engineering EPFL-ISIC-LSCI

$\mathrm{CH}-1015$ Lausanne

Tel.: +41216939781

Fax: +41216939305

E-mail: xile.hu@epfl.ch

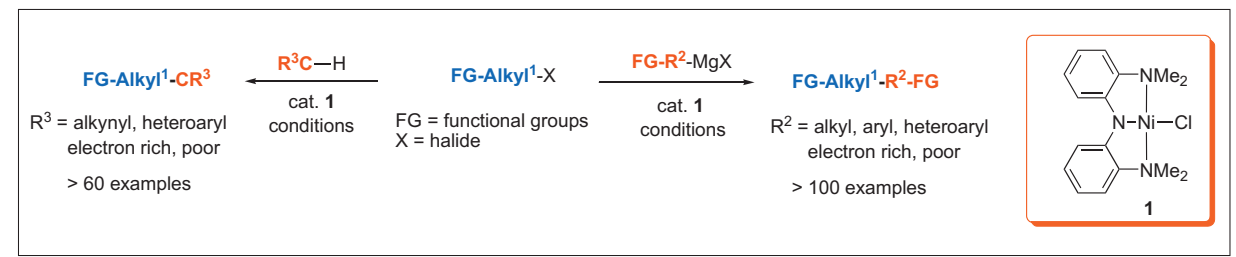

Scheme 1. The pincer catalyst $\left[\left(\mathrm{Me}_{2} \mathrm{~N}\right) \mathrm{NiCl}\right]$ and the reactions it catalyzes.

'privileged ligands' in homogeneous catalysis. ${ }^{[1]}$ Nitrogen donors are often said to be 'hard',[2] and therefore would match well the 'hard' first-row transition metal fragments. The combination of these two factors might lead to interesting chemistry. Having this in mind, we developed the synthesis of the ligand and its coordination chemistry. ${ }^{[3]}$ We decided to focus on $\mathrm{Ni}$ because its complexes were easy to make and characterize (crystalline and diamagnetic).

When exploring the reactivity of ${ }^{\mathrm{Me}} \mathrm{N}_{2} \mathrm{~N}-\mathrm{Ni}$ complexes, we found that complex $1^{[4]}$ mediated stoichiometric $\mathrm{C}-\mathrm{C}$ coupling of non-activated alkyl halides. ${ }^{[3,5]}$ Whereas cross coupling of aryl and vinyl electrophiles is now well developed, coupling of non-activated alkyl electrophiles is challenging due to unproductive $\beta-\mathrm{H}$ elimination. ${ }^{[6,7]}$ The activity of $\mathbf{1}$ prompted us to explore its application in catalytic coupling reactions. Indeed, complex $\mathbf{1}$ was an excellent catalyst for the coupling of non-activated alkyl halides with alkyl and aryl Grignard reagents. The catalysis displays broad scope and high functional group tolerance. More than 100 examples have been demonstrated (Scheme 1). ${ }^{[8,9]}$ Ester, amide, keto, ether, thioether, acetal, nitrile, various heterocyclic groups, and even aryl halide units are well tolerated. The scope is unusual for coupling reactions using Grignard reagents as nucleophiles, and underscores the high activity of the Ni catalyst.

We have attempted to replace the nucleophiles from reactive Grignard reagents to compounds containing weakly acidic $\mathrm{C}-\mathrm{H}$ bonds (Scheme 1). Indeed we found methods to couple alkyl halides directly with terminal alkynes and aromatic heterocycles. ${ }^{[10,11]}$ These reactions avoided the use of organometallic reagents. They are straightforward, atom- and step-economic, and have high group tolerance.

We have also started to study the mechanism of catalysis. For example, we found that $\beta-\mathrm{H}$ elimination is kinetically accessible but thermodynamically uphill in Ni-alkyl complexes of ${ }^{\mathrm{Me}} \mathrm{N}_{2} \mathrm{~N}$. [12] This work reveals one of the main factors that contribute to the efficiency of $\mathbf{1}$ in catalysis.

A large part of our work in Ni catalysis has been summarized in an earlier account in CHIMIA. ${ }^{[13]}$ Interested readers are referred to that account for more details.

\section{Bio-mimetic Chemistry of [Fe]- Hydrogenase}

A second project of the group is the model chemistry of [Fe]-hydrogenase. Hydrogenases are enzymes that catalyze $\mathrm{H}_{2}$ production and utilization in nature. The functions of hydrogenases depend on inexpensive and abundant metals such as $\mathrm{Fe}$ and Ni. ${ }^{[14,15]}$ The structures of the active sites of hydrogenases and the mechanisms by which they produce and activate $\mathrm{H}_{2}$ are of great interest, both fundamentally and as biological models for the development of efficient $\mathrm{H}_{2}$-processing catalysts. 


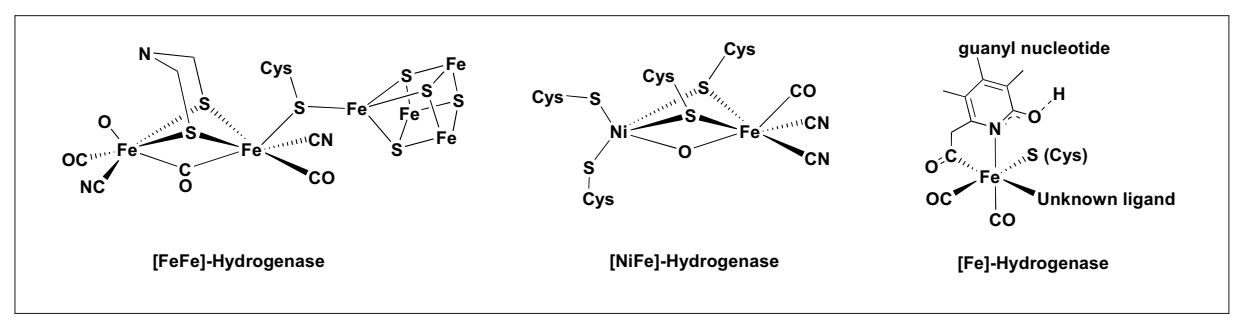

Fig. 1. The active sites of three different hydrogenases.

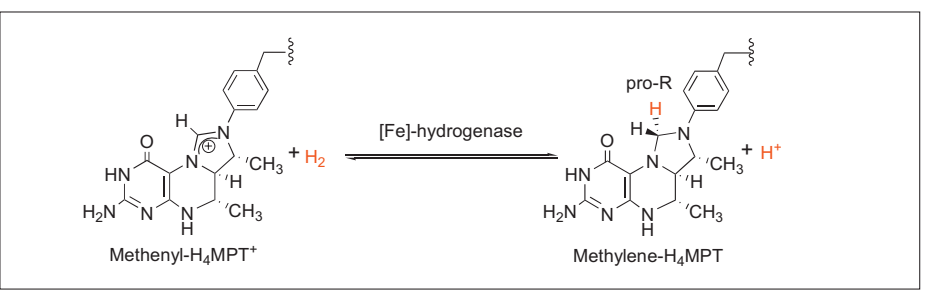

Scheme 2. The reaction catalyzed by [Fe]-hydrogenase. In this reaction, a hydride is stereospecifically transferred from $\mathrm{H}_{2}$ into the pro- $\mathrm{R}$ position of methylenetetrahydromethanopterin with a $\Delta \mathrm{G}^{\mathrm{o}^{\prime}}$ of $-5.5 \mathrm{~kJ} / \mathrm{mol}$.

Three types of hydrogenases are known: the $[\mathrm{NiFe}]$-hydrogenase, the [FeFe]-hydrogenase, and the $\mathrm{H}_{2}$-forming methylenetetrahydromethanopterin dehydrogenase (Hmd) which is now called [Fe]-hydrogenase (Fig. 1). ${ }^{[14,15]}$ The [Fe]hydrogenase, found only in some methanogenic archaea growing on $\mathrm{H}_{2}$ and $\mathrm{CO}_{2}$, catalyzes the reversible reduction of methenyltetrahydromethanopterin (methenyl- $\mathrm{H}_{4} \mathrm{MPT}^{+}$) with $\mathrm{H}_{2}$ to form methylenetetrahydromethanopterin (methylene$\mathrm{H}_{4} \mathrm{MPT}$ ) and $\mathrm{H}^{+}$(Scheme 2). This reaction is an intermediary step in the reduction of $\mathrm{CO}_{2}$ to methane by methanogens grown under nickel-limiting conditions. ${ }^{[15,16]}$

$[\mathrm{Fe}]$-hydrogenase is a recent discovery and its crystal structure was first reported in $2008^{[17]}$ and re-interpreted in 2009. [18] The 2008-proposed active site was shown in Fig. 2, left. ${ }^{[17]}$ The Fe center is 5- or 6-coordinate depending on the state of the enzyme. The most interesting feature is the mono-dentate pyridone ligand with noncoordinating carboxylic acid and pyridone oxygen donors. Shortly after, the structure of C176A mutated [Fe]-hydrogenase crystallized in the presence of dithiothreitol was solved. ${ }^{[18]}$ The result led to a new interpretation of the active site in the wild type [Fe]-hydrogenase. The revised structure (2009) consists of a Fe center coordinated to the pyridone cofactor through both pyridinyl nitrogen and acyl carbon atoms (Fig. 2 , right).

We began to develop model complexes for the active site of [Fe]-hydrogenase following the guide of the 2008 structure. We first focused on the unusual pyridone ligand found in the enzyme. Prior to this work, there were no known complexes in which a pyridone ligand coordinates to a single Fe center. Employing the procedure outlined in Scheme 3, we were able to make the first iron bis(carbonyl)pyridonate complex (2). ${ }^{[19]}$ The IR and Mössbauer parameters of this complex are similar to those of the enzyme.

After the 2009 structure of [Fe]hydrogenase was reported, we turned our attention to model the acyl ligand of the enzyme. An $\mathrm{Fe}^{\mathrm{II}}$ acyl precursor complex 3 was prepared by reaction of MeLi with $\mathrm{Fe}(\mathrm{CO})_{5}$ and then oxidation with $\mathrm{I}_{2} .3$ reacted with sodium 6-methyl-2-mercaptopyridinate to give the diiron dithiolate complex 4, which reacted rapidly with phosphine, isocyanide, and cyanide to form 6-coordinate and mononuclear $\mathrm{Fe}^{\mathrm{II}}$ complexes 5-7 (Scheme 4). ${ }^{[20]}$ Compared to the active site of [Fe]-hydrogenase (Fig.

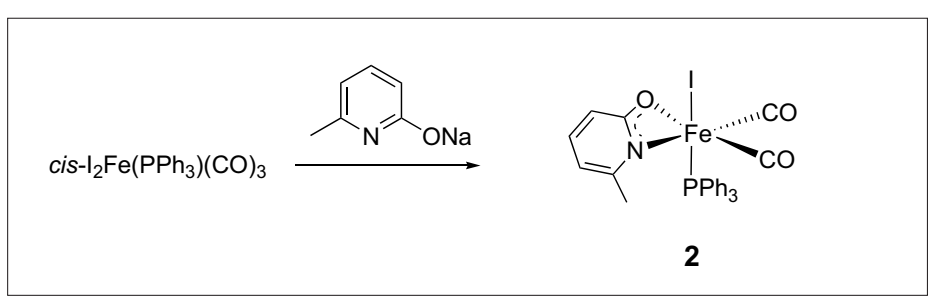

Scheme 3. Synthesis of an iron bis(carbonyl) pyridonate complex.

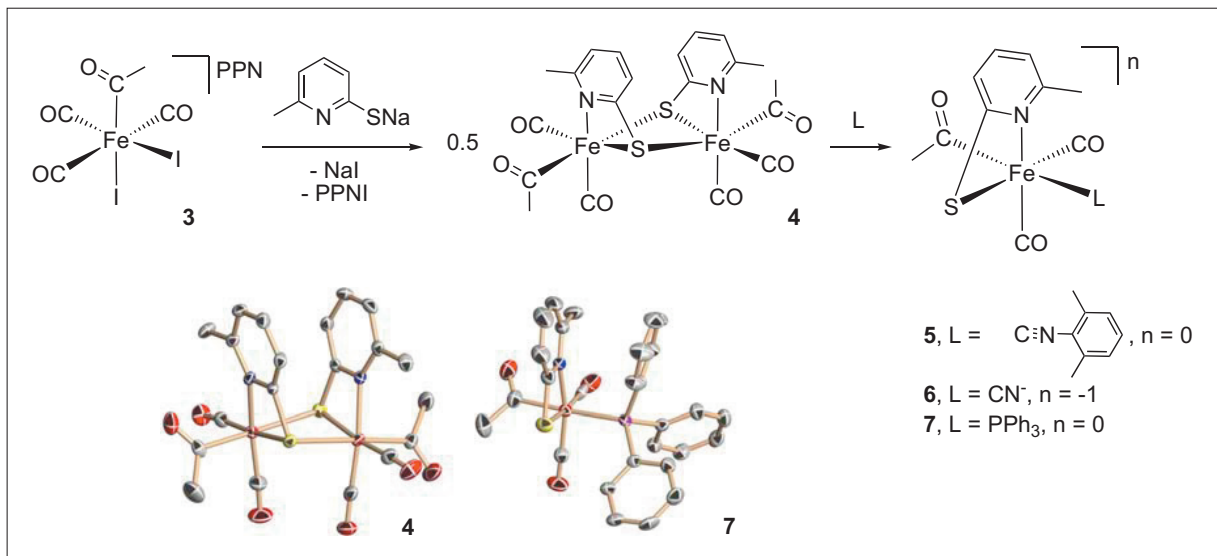

Scheme 4. Synthesis of iron acyl model complexes 5-7, and structures of $\mathbf{4}$ and $\mathbf{7}$. 


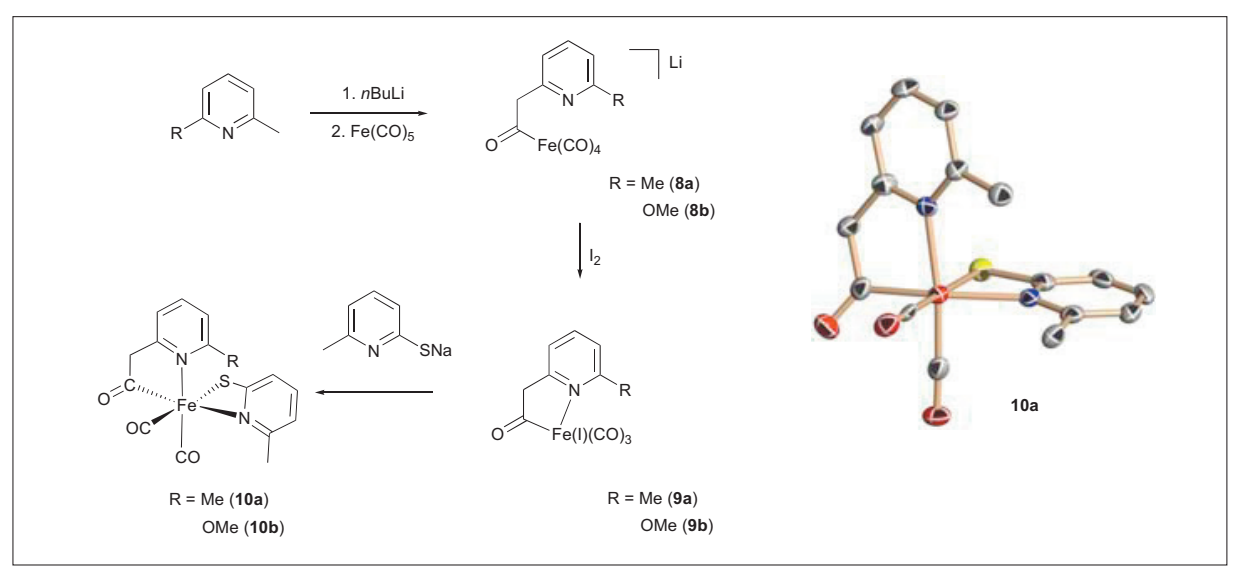

Scheme 5. Synthesis of iron pyridinyl methal acyl model complexes $10 \mathbf{a}$ and $10 \mathbf{b}$, and structure of 10a.

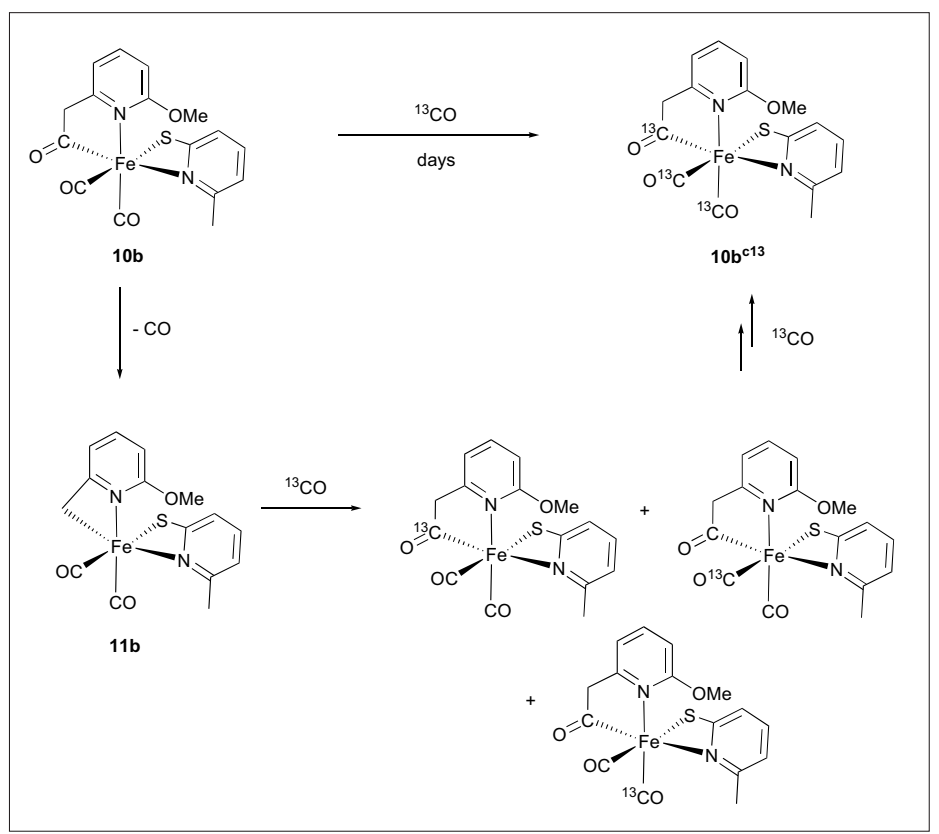

Scheme 6. CO exchange reaction of mononuclear model complex 10b.
[11] O. Vechorkin, V. Proust, X. L. Hu, Angew. Chem., Int. Ed. 2010, 49, 3061.

[12] J. Breitenfeld, O. Vechorkin, C. Corminboeuf, R. Scopelliti, X. L. Hu, Organometallics 2010, $29,3686$.

[13] X. L. Hu, Chimia 2010, 64, 231.

[14] P. M. Vignais, B. Billoud, J. Meyer, FEMS Microbiol. Rev. 2001, 25, 455.

[15] S. Shima, R. K. Thauer, Chem. Rec. 2007, 7, 37.

[16] R. K. Thauer, A. R. Klein, G. C. Hartmann, Chem. Rev. 1996, 96, 3031

[17] S. Shima, O. Pilak, S. Vogt, M. Schick, M. S. Stagni, W. Meyer-Klaucke, E. Warkentin, R. K. Thauer, U. Ermler, Science 2008, 321, 572.

[18] T. Hiromoto, K. Ataka, O. Pilak, S. Vogt, M. S. Stagni, W. Meyer-Klaucke, E. Warkentin, R. K. Thauer, S. Shima, U. Ermler, FEBS Lett. 2009, $583,585$.

[19] B. V. Obrist, D. F. Chen, A. Ahrens, V. Schunemann, R. Scopelliti, X. L. Hu, Inorg. Chem. 2009, 48, 3514.

[20] D. F. Chen, R. Scopelliti, X. L. Hu, J. Am. Chem. Soc. 2010, 132, 928.

[21] D. F. Chen, R. Scopelliti, X. L. Hu, Angew. Chem., Int. Ed. 2010, 49, 7512.
With a large number of model complexes in hand, we have started to explore their reactivity. We found that complex 4 reacted with $\mathrm{CO}$ to form a mononuclear $\mathrm{Fe}$ tris(carbonyl) complex, which is a mimic of the CO-inhibited [Fe]-hydrogenase. ${ }^{[21]}$ We also found that complex $\mathbf{9 b}$ undergoes ${ }^{13} \mathrm{CO}$ exchange under one atmosphere of ${ }^{13} \mathrm{CO}$ gas. We propose that one key step is the elimination of $\mathrm{CO}$ from the acyl ligand to form a Fe-alkyl species (11b), which reacts with ${ }^{13} \mathrm{CO}$ to incorporate the first ${ }^{13} \mathrm{C}$ element (Scheme 6). Repeating the same process two more times would lead to the

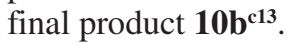

Currently we are working on the $\mathrm{H}_{2}$ activation chemistry using the model compounds.

\section{Acknowledgement}

I would like to thank my co-workers for their lab-work and intellectual contributions. Their names appear in the relevant group publications cited in this account. Our work has been supported by the EPFL and the Swiss National Science Foundation (project no. 200021_119663 and 200021_126498).

Received: April 4, 2011

[1] 'The chemistry of pincer compounds', Eds D. Morales-Morales, C. M. Jensen, Elsevier, Amsterdam, 2007.

[2] R. J. Pearson, J. Am. Chem. Soc. 1963, 85, 3533.

[3] Z. Csok, O. Vechorkin, S. B. Harkins, R. Scopelliti, X. L. Hu, J. Am. Chem. Soc. 2008, 130, 8156.

[4] Complex $\mathbf{1}$ is now commercially available from Sigma-Aldrich bearing the synonym 'Nickamine'. The name reflects the combination of nickel and amine ligands.

[5] O. Vechorkin, Z. Csok, R. Scopelliti, X. L. Hu, Chem.-Eur. J. 2009, 15, 3889.

[6] M. R. Netherton, G. C. Fu, Adv. Synth. Catal. 2004, 346, 1525.

[7] A. C. Frisch, M. Beller, Angew. Chem., Int. Ed. 2005, 44, 674

[8] O. Vechorkin, X. L. Hu, Angew. Chem., Int. Ed. 2009, 48, 2937.

[9] O. Vechorkin, V. Proust, X. L. Hu, J. Am. Chem Soc. 2009, 131, 9756.

[10] O. Vechorkin, D. Barmaz, V. Proust, X. L. Hu, J. Am. Chem. Soc. 2009, 131, 12078. 